\title{
Connaissances, attitudes et pratiques du personnel soignant sur l'hépatite virale $B$ au Centre Hospitalier Régional Lomé Commune en 2018.
}

\section{Knowledge, Attitudes and Practices of caregivers on viral hepatitis B at Lomé Commune Regional Hospital in 2018.}

Bawè LD ${ }^{1}$, Agbéko $F^{2}$, Kotosso $\mathrm{A}^{1,3}$, Némi $\mathrm{KD}^{4}$, Abaltou $\mathrm{B}^{1,3}$, Moukaïla $\mathrm{AR}^{1}$, Patassi $\mathrm{AA}^{1}$, Watéba $\mathrm{MI}^{1}$.

1 : Service des Maladies Infectieuses et Tropicales, CHU Sylvanus Olympio, Lomé (Togo) ;

2 : Centre Hospitalier Régional Lomé-Commune ;

3: Centre Hospitalier des Armées de Lomé;

4: Service de Médecine Interne, CHU SylvanusOlympio, Lomé (Togo)

Auteur correspondant : Bawè Lidaw Déassoua ; téléphone : 0022890907030 ; Email :

alainbawe@yahoo.fr

\section{Résumé}

Objectifs: Décrire les connaissances, attitudes et pratiques du personnel soignant du Centre Hospitalier Régional Lomé Commune par rapport au risque de contamination etcomplications de l'hépatite viral B.

Patients et méthodes : Il s'est agi d'une étude transversale, descriptive à visée analytique de type Connaissances, Attitudes et Pratiques qui s'est déroulée au Centre Hospitalier Régional Lomé Commune.

Résultats : Le taux de participation à cette enquête était de $76 \%$ avec une prédominance masculine $50,68 \%)$. Les catégories professionnelles les plus représentées étaient les techniciens supérieurs de santé $(32,2 \%)$, les infirmiers $(28,1 \%)$ et les sages-femmes $(17,8 \%)$ avec une moyenne d'âge de 36 ans (25-59 ans). L'étiologie virale de l'hépatite B était connue dans $96,6 \%$ des cas et les voies de transmission sanguine, sexuelle et mère-enfant ont été citées dans respectivement 94,52\%, 78,08\% et $74,66 \%$. Les médecins maîtrisaient de façon significative les signes évocateurs de l'hépatite virale B que les sages-femmes et les infirmiers $(p=0,019)$. Le ténofovir était connu dans $52,7 \%$ comme molécule utilisée dans le traitement de l'hépatite B et la vaccination a été évoquée comme moyen de prévention dans $98,63 \%$. Seulement un tiers des enquêtés $(36,99 \%)$ demandaient la sérologie de l'hépatite virale Ben cas d'accident d'exposition au sang. Le taux de couverture vaccinale du personnel soignant était de $65,10 \%$. Les niveaux de connaissances, d'attitudes et de pratiques étaient significativement meilleurs chez les sages-femmes $(\mathrm{p}<0,005)$.

Conclusion : La faible couverture vaccinale du personnel soignant impose des actions de formations continues et de sensibilisation couplées d'une subvention de vaccination.

Mots - clés : Hépatite B, connaissances, soignants, Togo.

\section{Abstract}

Objectives: Describe knowledge, attitudes and practices of Lomé Commune Regional Hospital caregivers with regard to viral hepatitis B contamination risk and complications.

Patients and Methods: This was Knowledge, Attitudes and Practices based cross-sectional, descriptive study with an analytical aim which took place at Lomé Commune Regional Hospital.

Results: The participation rate in this survey was $76 \%$ with a male predominance of $50.68 \%$ ). The most represented professional categories were Senior Health Technicians (32.2\%), Nurses $(28.1 \%)$ and Midwives (17.8\%) with an average age of 36 years (25-59 years). The viral etiology of hepatitis B was known in $96.6 \%$ of the cases and blood, sexual and mother-to-child transmission routes were cited in $94.52 \%, 78.08 \%$ and $74.66 \%$ of the participants respectively. Physicians significantly knew better about signs suggestive of viral hepatitis B than Midwives and Nurses did $(p=0.019)$. Tenofovir was known in $52.7 \%$ as a molecule used in the treatment of hepatitis B and immunization was mentioned as a means of prevention in $98.63 \%$ of cases. Only one third of the respondents (36.99\%) asked for viral hepatitis B serology in case of an accidental blood exposure. Caregivers' immunization coverage rate was $65.10 \%$. Levels of knowledge, attitudes and practices were significantly better among midwives $(\mathrm{p}<0.005)$.

Conclusion: Caregivers' low immunization coverage requires continuing education and awareness raising activities coupled with a vaccination subsidy. 
Key words : Hepatitis B, knowledge, caregivers, Togo.

\section{Introduction}

L'hépatite virale B est un problème majeur de santé publique qui appelle une réponse de toute urgence [1]. En 2015, le nombre des personnes atteintes d'une infection chronique par le virus de l'hépatite B (VHB) était de 257 millions et a causé 1,34 millions de décès, soit un nombre comparable à celui des décès dus à la tuberculose et supérieur aux décès causés par le VIH [1]. La prévalence de l'hépatite $B$ est la plus élevée en Afrique subsaharienne et en Asie de l'Est, où entre 5 et $10 \%$ de la population adulte est atteinte d'hépatite virale chroniqueB. La transmission mère-enfant du virus de l'hépatite B est un mode de transmission fréquent dans les milieux de forte prévalence [2].Au Togo, aucune prévalence globale de l'hépatite B n'a été estimée auparavant, mais des études parcellaires ont permis d'avoir des prévalences sectorielles. On note une séroprévalence de 16,36 \% (189/1155) au sein de la population générale de Lomé la capitale ; plus élevée dans la tranche d'âge de o à 5 ans pour le VHB [3] contre une prévalence de 19,8\% dans une population âgée de $20-39$ ans $(\mathrm{n}=1200)$ [4]. Chez les jeunes de 15 à 24 ans, la prévalence de l'antigène HBs était de $16,4 \%$. $(n=2101)$ [5].Le risque de contamination en milieu hospitalier se présente sous de multiples aspects, notamment : matériels, comportementaux, organisationnels et culturels [6]. Le risque infectieux viral secondaire aux accidents d'exposition aux liquides biologiques constitue une menace pour la santé des professionnels de soins dans les pays en développement [7]. Ce risque est encore insuffisamment pris en compte au Togo [8]. Hôpital de référence depuis 2010, le Centre Hospitalier Régional Lomé Commune (CHR-LC) n'a pas encore connu une telle évaluation. L'objectif principal de cette étude était de décrire les connaissances, attitudes et pratiques du personnel soignant du CHR-LC par rapport au risque de contamination et decomplication de l'hépatite viral B.

\section{Matérielet méthodes}

Il s'est agi d'une étude transversale, descriptive à visée analytique de type Connaissances, Attitudes et Pratiques qui s'est déroulée du 18 décembre 2017 au 16 mars 2018, soit une période de trois mois au CHR-LC.Ont été inclus les prestataires impliqués directement dans les activités de soins et donc plus exposés au risque de contamination du virus de l'hépatite B par leur contact avec le sang et liquides biologiques (médicaux, chirurgicaux, gestion des déchets médicaux).L'ensemble du personnel soignant (plus exposé au contact du sang et liquides biologiques) impliqué directement dans les activités de soins a été concerné. La population cible était de 192.Les données ont été recueillies à partir d'un questionnaire préalablement prétexté. $\mathrm{Ce}$ questionnaire apermis de recueillir les réponses du personnel sur leurs connaissances, attitudes et pratiques de l'hépatite virale $\mathrm{B}$ face à certaines situations du vécu hospitalier quotidien ; en plus de leurs caractéristiques sociodémographiques (âge, sexe, profil, niveau d'instruction, situation matrimoniale, expérience professionnelle).Les données ont été saisies et analysées avec le logiciel Epi-Info version 3.5.4.Le test de Khi- deux a été utilisé pour comparer les pourcentages. Le seuil de signification de $5 \%$ a été considéré (seuil $\mathbf{p}_{\mathbf{0}}=\mathbf{0 , 0 5}$ ). La différence a été considéré comme significative si $\mathbf{p}_{\mathbf{c}}$ ( $\mathrm{p}$ calculé) est inférieur à po. Les tableaux et les figures ont été réalisés à l'aide des logiciels Microsoft Excel et Word.Une autorisation du directeur du CHR-LC a été préalablement obtenue avant le début cette étude dans les services de soinsafin de recueillir les données dans l'anonymat.

\section{Résultats}

\section{Caractéristiques socio-démographiques}

Le taux de participation global était de 76\%; 146 agents ont répondu au questionnaire sur les 192 personnels soignants de la structure.On note une prédominance masculine $(51 \%)$ avec un sex ratio Homme/Femme de 1,03. L'âge moyen du personnel était de 36 ans $\pm 8,48$ (extrêmes de 25- 59 ans). L'ancienneté dans la profession médicale était en moyenne de 8 ans avec un écart-type de 6,3 et des extrêmes de 0 - 29 ans.Les techniciens supérieurs de santé et les infirmiers étaient les plus représentés et la majorité du personnel provenait du pôle chirurgical (Tableau I).

Tableau I : Répartition du personnel enquêté selon le sexe, la catégorie professionnelle et le pôle d'activité(n=146).

\begin{tabular}{lcc}
\hline Paramètres & Effectif $(\mathbf{N})$ & $\begin{array}{c}\text { Pourcentage } \\
(\mathbf{\%})\end{array}$ \\
\hline Sexe & & \\
\hline Homme & 74 & 51 \\
Femme & 72 & 49 \\
\hline Total & 146 & 100 \\
\hline Catégories & & \\
professionnelles & & \\
\hline TSS* & 47 & 32,2 \\
IDE**/IAE*** & 41 & 28,1 \\
Sage-Femme & 26 & 17,8 \\
Garde-malade & 16 & 10,95 \\
Médecin & 16 & 10,95 \\
\hline Total & 146 & 100 \\
\hline Pôle d'activité & & \\
\hline Chirurgical & 82 & 56,2 \\
Médical & 51 & 34,9 \\
Laboratoire & 13 & 8,9 \\
Total & 146 & 100 \\
\hline Age moyen (ans) [Extrême] & $\mathbf{3 6} \pm \mathbf{8 , 4 8}[\mathbf{2 5}-\mathbf{5 9 ]}$ \\
\hline *TSS : Technicien Supérieur de Santé $; * *$ IDE : Infirmier \\
Diplomé d'Etat ; **IAE: Infirmier Auxiliaire d'Etat
\end{tabular}




\section{Connaissances}

Définition de l'hépatite virale $B$ et dépistage

L'étiologie virale de l'atteinte du foie de l'hépatite B était connuepar la majorité du personnel $(96,60 \%)$. Le dosage de l'AgHBs a été cité dans $99,30 \%$ des cas pour le dépistage de l'infection.

Connaissance sur les voies de transmission, les signes évocateurs et les complications de l'hépatite virale $B$.

Le tableau II résume les différentes réponses du personnel de leurs connaissances sur les voies de transmission, les signes évocateurs et les complications de l'hépatite virale B.

Tableau II : Connaissances du personnel soignant surles voies transmission, les signes évocateurs, les complications, le traitement et la prévention( $\mathrm{n}=146)$.

\begin{tabular}{|c|c|c|c|}
\hline & Paramètres & $\mathbf{N}$ & $(\%)$ \\
\hline \multirow{7}{*}{ 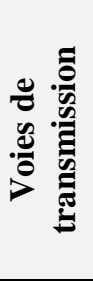 } & Sanguine & 138 & $(94,5)$ \\
\hline & Sexuelle & 114 & $(78,1)$ \\
\hline & Mère-enfant & 109 & $(74,7)$ \\
\hline & Orale & 17 & $(11,6)$ \\
\hline & Sueurs & 5 & $(3,4)$ \\
\hline & Larmes & 1 & $(0,7)$ \\
\hline & Ne sait pas & 2 & $(1,4)$ \\
\hline \multirow{7}{*}{ 晜 } & Ictère & 130 & $(89,0)$ \\
\hline & Hépatomégalie & 98 & $(67,1)$ \\
\hline & Asthénie & 88 & $(60,3)$ \\
\hline & Fièvre & 72 & $(49,3)$ \\
\hline & Arthralgies & 29 & $(19,9)$ \\
\hline & Troubles digestifs & 3 & $(2,1)$ \\
\hline & Urines foncées & 1 & $(0,7)$ \\
\hline \multirow{5}{*}{ ن } & Cirrhose & 114 & $(78,1)$ \\
\hline & Cancer du foie & 97 & $(66,4)$ \\
\hline & Hépatite chronique & 79 & $(54,1)$ \\
\hline & Hépatomégalie & 53 & $(36,3)$ \\
\hline & Ne sait pas & 2 & $(1,37)$ \\
\hline \multirow{7}{*}{ } & Ténofovir & 77 & $(52,7)$ \\
\hline & Interféron & 32 & $(21,6)$ \\
\hline & Lamivudine & 28 & $(18,9)$ \\
\hline & Entécavir & 4 & $(2,7)$ \\
\hline & Chirurgie & 2 & $(1,4)$ \\
\hline & Bonne hygiène & 2 & $(1,4)$ \\
\hline & Ne sait pas & 2 & $(1,4)$ \\
\hline \multirow{5}{*}{ 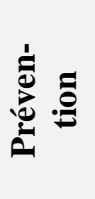 } & Vaccination & 144 & $(98,6)$ \\
\hline & Barrière de protection & 93 & $(63,7)$ \\
\hline & Stérilisation & 83 & $(56,8)$ \\
\hline & IEC & 82 & $(56,2)$ \\
\hline & Suivi de grossesse & 1 & $(0,7)$ \\
\hline
\end{tabular}

En dehors de la voie de transmission sanguine, sexuelle et la transmission mère-enfant qui ont été citées majoritairement, d'autres voies de transmissions ont été évoquées (tableau III).
Tableau III : Répartition du personnel enquêté sur la connaissance des voies de transmission, les signes évocateurs et les complications de l'hépatite virale $B(n=146)$.

\begin{tabular}{llc}
\hline & Paramètres & $\mathbf{N}(\%)$ \\
\hline & Sanguine & $138(94,52)$ \\
& Sexuelle & $114(78,08)$ \\
Voies de & Mère-enfant & $109(74,66)$ \\
transmission & Orale & $17(11,64)$ \\
& Sueurs & $5(3,42)$ \\
& Larmes & $1(0,68)$ \\
& Ne sait pas & $2(1,37)$ \\
\hline \multirow{5}{*}{ Signes } & Ictère & $130(89,04)$ \\
évocateurs & Hépatomégalie & $98(67,12)$ \\
& Asthénie & $88(60,27)$ \\
& Fièvre & $72(49,32)$ \\
& Arthralgies & $29(19,86)$ \\
& Troubles & $3(2,05)$ \\
& digestifs & $1(0,68)$ \\
\hline \multirow{4}{*}{ Complications } & Urines foncées & $114(78,08)$ \\
& Cirrhose & $97(66,44)$ \\
& Cancer du foie & $79(54,11)$ \\
& Hépatite & \\
& chronique & $53(36,3)$ \\
& Hépatomégalie & $2(1,37)$ \\
\hline & Ne sait pas &
\end{tabular}

Connaissances sur le traitement et la prévention Le ténofovir a été cité comme molécule utilisée pour le traitement de l'hépatitevirale $B$ et la vaccination a été reconnue comme moyen de prévention par la majorité du personnel(tableau IV).

Tableau IV : Répartition du personnel selon la connaissance sur le traitement et la prévention de l'hépatite virale $\mathrm{B}(\mathrm{n}=146)$.

\begin{tabular}{llc}
\hline Paramètres & & $\mathbf{N}(\%)$ \\
\hline & Ténofovir & $77(52,7)$ \\
& Interféron & $32(21,56)$ \\
& Lamivudine & $28(18,92)$ \\
& Entécavir & $4(2,7)$ \\
& Chirurgie & $2(1,35)$ \\
& Bonne hygiène & $2(1,35)$ \\
& Ne sait pas & $2(1,35)$ \\
\hline \multirow{5}{*}{ Prévention } & Vaccination & $144(98,63)$ \\
& Barrière & $93(63,7)$ \\
& protection & \\
& Stérilisation & $83(56,85)$ \\
& IEC & $82(56,16)$ \\
& Suivi grossesse & $1(0,68)$ \\
\hline
\end{tabular}

La moitié des enquêtés avaient $(50,70 \%)$ affirmé qu'il existait un traitement contre l'hépatite virale B. Le Ténofovir avait été évoqué par la moitié des enquêtés $(52,70 \%)$ comme médicament utilisé pour le traitement de l'hépatite virale $B$.

Connaissances, niveau d'instruction des soignants et anciennetéprofessionnelle 
Globalement, le niveau de connaissances sur l'HVB des soignants de niveau universitaire étaitsignificativement supérieurs $(p<0,05)$. En particulier, la différence était marquée sur la connaissance des signes évocateurs et des voies de transmission (Tableau V).

Tableau V: Répartition du personnel selon la connaissance sur les signes évocateurs, les voies de transmission de l'hépatite virale B selon le niveau d'instruction et l'ancienneté professionnelle $(n=146)$.

\begin{tabular}{|c|c|c|c|c|c|c|}
\hline & & \multicolumn{2}{|c|}{ Bonne } & \multicolumn{2}{|c|}{ Mauvaise } & \multirow[t]{2}{*}{$\mathbf{p}$} \\
\hline & & $\mathrm{N}$ & $\%$ & $\mathrm{~N}$ & $\%$ & \\
\hline & & \multicolumn{5}{|c|}{ Signes évocateurs } \\
\hline \multirow{3}{*}{ 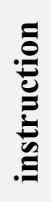 } & $\mathrm{BAC}+$ & 79 & 67,5 & 38 & 32,5 & \multirow[t]{2}{*}{0,003} \\
\hline & $<$ BAC & 11 & 37,9 & 18 & 62,1 & \\
\hline & & \multicolumn{5}{|c|}{ Voies de transmission } \\
\hline \multirow{2}{*}{$\begin{array}{l}\bar{Z} \\
\bar{z} \\
\bar{Z} \\
\bar{Z}\end{array}$} & $\mathrm{BAC}+$ & 94 & 79,7 & 24 & 20,3 & \multirow[t]{2}{*}{0,004} \\
\hline & $<\mathrm{BAC}$ & 15 & 53,6 & 13 & 46,4 & \\
\hline \multirow{2}{*}{ } & $<5$ ans & 49 & 72,1 & 19 & 27,9 & \multirow[t]{2}{*}{0,034} \\
\hline & $>5$ ans & 43 & 55,1 & 35 & 44,9 & \\
\hline
\end{tabular}

\section{Connaissances et catégories professionnelles}

Les médecins maîtrisaient de façon statistiquement significative les signes évocateurs de l'hépatite virale $\mathrm{B}$ que les sages-femmes et les infirmiers ( $\mathrm{p}=$ 0,019).

Attitudes

Moins de la moitié des enquêtés ont affirmé demander systématiquement l'AgHBs à leurs patients $(41,03 \%)$.Les principales raisons de la faible demande systématique du dépistage étaient : l'absence de signes évocateurs $(41,77 \%)$, examen non obligatoire $(21,52 \%)$, le coût élevé des examens pour le dépistage $(16,46 \%)$ et la rupture de réactif $(12,66 \%)$.

Devant un nouveau-né issu d'une mère AgHBs positif, moins de la moitié affirmait que le nouveauné devrait bénéficierdu vaccin contre le VHB et éventuellement de l'immunoglobuline anti hépatite B à la naissance $(43,84 \%)$.

Dépistage systématique de l'hépatite virale B Moins de la moitié des enquêtés ont affirmé demander systématiquement l'AgHBs à leurs patients $(41,03 \%)$. Les causes de la faible demande systématique de dépistage sont principalement dues à l'absence de signes évocateurs $(41,77 \%)$, du fait que l'examen n'est pas obligatoire $(21,52 \%)$ et du coût élevé des examens pour ledépistage (16,46\%).

Conduite à tenir devant un nouveau-né de mère infectée par l'hépatite virale $B$
Moins de la moitié des enquêtés affirmaient que les nouveau-nés de mère ayant l'AgHBs positif bénéficiaient du vaccin $(57,53)$ et éventuellement de l'immunoglobuline anti hépatite $\mathrm{B}$ à la naissance $(43,84 \%)$. L'éviction de l'allaitement maternel a été citée par7,53\% des participants.

Niveau d'instruction, pôle d'activité, ancienneté professionnelle et catégorie professionnelle; et attitudes.

Il y avait des différences significatives dans les attitudes de prise de décision et de demande systématique du dépistage selon le niveau d'instruction, la catégorie professionnelle et le pôle d'activité (Tableau VI).

Tableau VI : Répartition du personnel selon les attitudes de prise de décision selon le niveau d'instruction et pôle d'activité ; et les attitudes de demande systématique du dépistage de l'hépatite B selon l'ancienneté professionnelle et la catégorie professionnelle $(n=146)$.

\begin{tabular}{|c|c|c|c|c|c|}
\hline & \multicolumn{2}{|c|}{ Bonne } & \multicolumn{2}{|c|}{ Mauvaise } & \multirow[t]{2}{*}{$\mathbf{P}$} \\
\hline & $\mathrm{N}$ & $\%$ & $\mathrm{~N}$ & $\%$ & \\
\hline \multicolumn{6}{|c|}{ Prise de décision } \\
\hline ๘ & 93 & 78,8 & 25 & 21,2 & \multirow{2}{*}{0,000} \\
\hline 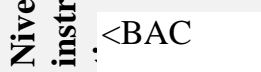 & 9 & 32,1 & 19 & 67,9 & \\
\hline ¿্ন $\quad$ Médical & 43 & 86 & 7 & 14 & \multirow{2}{*}{0,044} \\
\hline 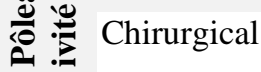 & 58 & 70,7 & 24 & 29,3 & \\
\hline \multicolumn{6}{|c|}{ Demande systématique } \\
\hline 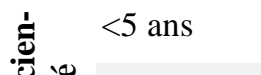 & 34 & 51,5 & 32 & 48,5 & \multirow[t]{2}{*}{0,0004} \\
\hline$\sum \stackrel{\Xi}{\Xi}>5$ ans & 14 & 21,9 & 50 & 78,1 & \\
\hline \multirow{2}{*}{ 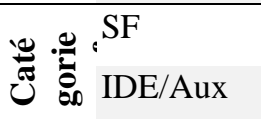 } & 22 & 84,6 & 4 & 15,4 & \multirow{2}{*}{0,000} \\
\hline & 11 & 31,4 & 24 & 68,6 & \\
\hline
\end{tabular}

\section{Pratiques}

Huit soignants sur dix avaient été dépistés positifs à l'AgHBs $(81,50 \%)$.

Les raisons du non dépistage de la proportion restante étaient la négligence, le coût élevé du dépistage et le manque d'information.La couverture vaccinale contrele VHB chez le personnel enquêté était de $65,10 \%$. Elle était plus faible chez les infirmiers $(45,7 \%)$ par rapport aux autres catégories professionnelles. Le coût élevé du vaccin, la négligence et le manque d'information sont les raisons évoquées par le personnel non vacciné.En cas d'accident d'exposition au sang et aux liquides biologiques, les gestes couramment pratiqués étaient le lavage au savon $(78,77 \%)$ et la désinfection $(74,66 \%)$. Seulement un tiers des enquêtés $(36,99 \%)$ demandaient la sérologie de l'hépatite virale B contre $60,27 \%$ pour la sérologie rétrovirale.

\section{Discussion}

Méthodologie 
Les connaissances, attitudes et pratiques du personnel soignant du CHR-LC sur l'hépatite virale $\mathrm{B}$ ont été évaluées à l'aide d'un questionnaire qui n'a pas subi de validation transculturelle et a été utilisé dans sa version originale. Des précisions et des explications en langues dialectales n'ont pas été nécessaires lors de l'entretien individualisé ; ce questionnaire nous a paru adapté à notre enquête car toutes les personnes interrogées ont répondu sans difficulté. La représentativité de notre échantillon et le taux élevé des participants (76\%) ont permis d'accorder une certaine validité à nos résultats. Ce taux de participation s'est beaucoup amélioré comparé à une étude similaire réalisée au Togo mais qui concernait les hépatites virales $\mathrm{B}$ et C [8].

\section{Caractéristiques socio-démographiques}

Le personnel paramédical est beaucoup plus représenté dans la plupart des études sur la question avec une prédominance féminine[8-11]. La prédominance de la gentféminine dans la profession paramédicale est constatée de façon générale.Les études similaires se sont déroulées chez des sujets relativement jeunes en ce qui concerne les âges moyens variant entre 29,5 et 39,5 ans $[7,9-11]$ avec une ancienneté dans la profession médicale inférieure à 10 ans dans la majorité des cas $[10,11]$. Dans un cas, les élèves infirmiers en formation et en stage dans les formations sanitaires au moment du déroulement de l'étude ont été pris en compte ; expliquant ainsi l'âge moyen de 29,5 ans très inférieur à la présente série [9].

\section{Connaissances et attitudes}

Dans l'ensemble, le niveau de connaissance du personnel soignant de notre série sur l'hépatite est B acceptable ; en ce qui concerne l'origine virale, les voies de transmission, les signes évocateurs, les complications et les moyens de prévention. La transmission verticale du virus de l'hépatite B semble être moins bien connue surtout par le personnel soignant paramédical $(42,3 \%)$ quel que soit l'ancienneté professionnelle comme le prouve une étude en Côte d'Ivoire [12] alors que ce mode de transmission était reconnue être le principal mode jusqu'en 1998 en Côte d'Ivoire (32,8\%)[13]. Plus encore dans une étude prenant en compte le personnel médical et paramédical $(n=74)$ ce mode transmission est peuconnu du personnel soignant soit 28,4\% (15).La méconnaissance de cette voie de transmission peut s'expliquer par l'absence d'enseignement consacré essentiellement aux hépatites virales en général, et l'hépatite virale B en particulier dans les écoles paramédicales.Le bon niveau de connaissances sur la question n'est pas suivi de bonnes attitudes et de bonnes pratiques du personnel soignant. Bien qu'informé du risque et des complications de l'hépatite $B$, une faible demande du dépistage est constatée dans la pratique ceci pour plusieurs raisons, entre autre le coût financier élevé. Au Togo, le dépistage coûte environ5500 francs CFA (environ 11 dollars américains) dans les formations sanitaires publiques ce qui n'est pas à la portée de la population générale qui a un revenu bas. Unefaible couverture vaccinale du personnel soignant est également retrouvée au Maroc (47,4\%) [7], 41\% au Sénégal [11] et $28 \%$ au Mali [14]. L'indisponibilité du vaccin (45\%), le manque d'information (38\%) et le coût élevé du vaccin (13\%) étaient les raisons évoquées ailleurs [11] ; tandis que le manque d'information $(43,5 \%)$ et le coût du vaccin représentaient les principales raisons évoquées dans une autre série [14].

\section{Conclusion}

Le niveau de connaissance du personnelsoignant est acceptable sur certains aspects de l'hépatite B notamment l'origine virale, les signes évocateurs, les principaux modes de transmission et les moyens de prévention ; par-contre les attitudes et pratiques restent insuffisantes et sont à renforcer.Avec cette faible couverture vaccinale, le personnel soignant reste exposé au risque de contamination du VHB. Il est donc important de multiplier les opportunités de formations continues et de sensibilisation du personnel soignant, couplées d'une subvention de vaccination.

\section{Conflitd'intérêt: Aucun Références}

1: World Health Organization. Global hepatitis report, 2017. World Health Organization, April 2017,83 p.

2: Organisation Mondiale de la Santé. Stratégie mondiale du secteur de la santé contre l'hépatite virale, 2016-2021. Vers une élimination de l'hépatite virale. Organisation mondiale de la santé, 2016, 55p.

3: Kolou M, Nadjir LK, Anyovi F, Katawa G, Abaltou B, Salou M. Séroprévalence deshépatites virales $\mathrm{B}$ et $\mathrm{C}$ au sein de la population générale de Lomé. J. Rech. Sci. Univ. Lomé(Togo), 2018, 20(1) : 297-305.

4 : Kolou M, Katawa G, Salou M, Gozo-Akakpo KS, Dossim S, Kwarteng A, Prince-David M. High Prevalence of Hepatitis B Virus Infection in the Age Range of 20-39 Years OldIndividuals in Lome. Open Virol J. 2017 Jan 12;11:1-7.

5 : Banla AK, Gani KT, Halatoko WA, Layibo Y, Akolly K, et al. (2015) Prevalence of the Surface Antigen of Hepatitis B Virus amongYouthAged 15 to 24 in TOGO in 2010. J Infect Dis TherInfectiousDiseases 3:238.

6 : Chevalier B, Margery J, Wade B. Perception du risque nosocomial parmi le personnel hospitalier de l'hôpital Principal de Dakar. Med Trop 2008 ; 68 : 593-6.

7 : Laraqui O, Laraqui S, Tripodi D, Ouazzani LC, Caubet A, Verger $\mathrm{C}$ et al. Evaluation des connaissances, attitudes et pratiques sur les 
hépatites virales $\mathrm{B}$ et $\mathrm{C}$ en milieu de soins au Maroc. Santé Publique 2009 ; 21 : 271-86.

8 : Bagny A, Bouglouga O, Djibril M, Lawson A, LaconiKaaga Y, Hamza Sama D et al. 9 : Mtengezo J, Lee H, Ngoma J, Kim S, Aronowitz T,DeMarco R, et al. Knowledge and attitudes toward HIV, hepatitis B virus, and hepatitis C virus infection amonghealth-care workers in Malawi. Asia Pac J OncolNurs 2016;3:344-51.

10: Khan KH, Ayub A, Hussain H, Qayyum I, Naseery AN. Knowledge attitude and practice regardinghepatitis B infection amonghospital staff nurses in HayatabadMedicalComplex, Peshawar. Khyber Med Univ J 2016; 8(2): 91-94.

11: Lawson ATD, Deme M, Diop-Nyafouna SA, Diop BM. Connaissances, attitudes et pratiques du personnel soignant du district sanitaire de RichardToll (Sénégal) en matière de dépistage de l'hépatite B. RAFMI 2017 ; 4 (2) : 26-29

12: Bouglouga O. Connaissances du virus de l'hépatite $B$ et pratiques en cas d'accident
Connaissances, attitudes et pratiques du personnel soignant sur le risque de transmission des hépatites virales B et $\mathrm{C}$ en milieu hospitalier au Togo. Med Sante Trop 2013 ; 23 : 300-3. d'exposition au sang du personnel soignant paramédical du CHU de Yopougon. Mémoire du Diplôme d'Etudes Spéciales d'Hépato-GastroEntérologie - Maladies de l'appareil digestif; $\mathrm{N}^{\circ}$ 2161, UFR Sciences Médicales Abidjan, 2014, 85p 13: Lohoues-Kouacou MJ, Touré M, Hillah J, Camara BM, N'Dri N, Kouamé KJ, Attia Y. Transmission in utero of the hepatitis $\mathrm{B}$ virus in IvoryCoast the case for mass vaccination. Sante.1998; 8(6):401-4

14 : Diallo M. Enquête connaissances, attitudes et pratiques du personnel soignant de l'Institutd'Ophtalmologie Tropicale de l'Afrique vis-à-vis de l'hépatite virale B. Thèse de Doctorat d'Etaten Médecine. Faculté de Médecine, de Pharmacie et d'Odontostomatologie. Université deBamako,

$73 \mathrm{p}$. 